Research Article

\title{
Prediction of Profile Deviation during Glass Molding of Double-Aspheric Lens
}

\author{
Zhu Kejun ${ }^{1,2, *}$, Shi Sheng ${ }^{1,2}$, Chen Zhigang ${ }^{1,2}$, Liu Zhihui ${ }^{1,2}$ and Zhang Yingying ${ }^{3}$ \\ ${ }^{1}$ College of Mechanical and Energy Engineering, Shaoyang University, Shaoyang, Hunan, 422000, China \\ ${ }^{2}$ Key Laboratory of Hunan Province for Efficient Power System and Intelligent Manufacturing, Shaoyang University, Hunan, 422000, \\ China \\ ${ }^{3}$ College of Optical Sciences, University of Arizona, Tucson, Arizona 85719, USA
}

Received 10 January 2020; Accepted 24 March 2020

\begin{abstract}
Glass molding technology has attracted considerable attention because of its low cost and high-volume production. However, the profile deviation mechanism of double-aspheric lens is currently unclear. A method of profile deviation prediction was developed in this study to reveal the influence law of molding parameters on the profile deviation of upper and lower aspheric surfaces. Based on the thermoviscoelastic constitutive model and finite element theory, a method of profile deviation prediction for the glass molding of double-aspheric lens was established. Single-factor analysis method was used to analyse the influence law of glass molding parameters, such as molding temperature, molding velocity, annealing rate, and holding pressure on profile deviation. Different influence laws of each molding parameter on the profile deviation of the upper and lower aspheric surfaces were discussed. Results demonstrate that the profile deviation decreases with the increase when the molding temperature lower than $570{ }^{\circ} \mathrm{C}$. When the molding temperature is above $570{ }^{\circ} \mathrm{C}$, the profile deviation slowly increases with the increase of molding temperature, and the profile deviation of the double-aspheric lens increases with the increase in the pressing velocity. The profile deviation of the upper and lower aspheric surface decreases as the annealing rate and the holding pressure increase, and the profile deviation of the upper surface is greater than the lower. The difference can be reduced by increasing the holding pressure. This study provides a reference for improving the molding accuracy of double-aspheric lens.
\end{abstract}

Keywords: Double-aspheric lens, Glass molding, Simulation prediction, Profile deviation

\section{Introduction}

The rapid development of optoelectronic communication, optics, automobile, bioengineering, aerospace technology, biotechnology, and life sciences has promoted the extensive use of optical component products [1-2]. Aspheric optical lens not only can effectively reduce image distortion and improve imaging quality but also can simplify the structure of the instrument; thus, this type of lens attracts considerable attention [3-4]. The traditional production of small-aperture aspheric glass lens uses a single removal processing method based on grinding and polishing; this approach has long production cycles and high processing costs [5-6]. Glass molding technology of glass lens is environmentally friendly, suitable for mass production, and characterized by low-cost and simple process; this technology has become the mainstream for manufacturing small-aperture aspheric glass lens [7].

We live in a complex world, because complex systems can be represented as networks, it is worthwhile analyzing the properties of networks as a way of understanding complex systems. Networks have many notable properties, such as the small-world property [3] and the scale-free property [4]. In recent years, network community structure property [5] has received much attention in diverse fields. In the academic domain, a network is generally denoted by a

\footnotetext{
*E-mail address: zhukejun1098@126.com

graph composed of nodes and edges. A community of a network is regarded as a subset of a graph. Often, communities are required to satisfy the condition that the nodes of a given community share similar features, while different communities have different node properties. The network community structure provides a microscopic perspective for understanding the functionality of a complex system. In the past few decades, a variety of methods have been proposed to detect community structures in networks. A survey can be found in [6].

During glass molding, the glass preform fills the mold cavity at high temperature and high pressure, replicates the profile of the mold, completes the required equal-volume deformation, and obtains the final aspheric lens with the designed shape through annealing and cooling [8]. The shape of the mold profile is usually processed depending on the aspheric curve of the designed lens. The actual profile curve of lens is slightly different from the originally designed profile curve due to the nonlinear variation of material properties, temperature and internal stress in the glass molding process, it decreases the molding accuracy and seriously affects the optical performance of molded lens [9].

Scholars have conducted simulation prediction of profile deviation of glass molding to reduce this deviation [10-12]. However, most of them have focused on single aspheric lens. The profile deviation and difference of the upper and lower profiles of aspheric lens have not been thoroughly studied. It is difficult to providing theoretical guidance and design support for the mass production of double-aspheric lens. 
Therefore, the urgent task is to accurately predict the profile deviation of double-aspheric surfaces and clarify the influence law of the upper and lower profile deviation.

Based on the thermoviscoelastic constitutive and heat conduction models, a finite element method was used to establish a simulation prediction model for the profile deviation of double-aspheric glass lens molding. The influence law of molding temperature, molding velocity, holding pressure, and annealing rate on aspheric profile deviation was determined. Moreover, the effects of the same glass molding parameters on the upper and lower aspheric profile deviations were investigated. The results provide a reference for enterprise production.

\section{Related background}

Scholars have thoroughly studied the properties of glass materials during glass molding. Saotome [13] performed glass molding experiments using two types of optical glass at $\mathrm{Tg}$ to $\mathrm{Tg}+30{ }^{\circ} \mathrm{C}$, obtained the relationship between stress and strain rates at different temperatures. However, thermoviscoelastic theory was not involved. Jain [9] used the Brillouin light scattering method to measure the elastic modulus of glass. Moreover, Newtonian fluid model was used to explain the deformation of glass at the molding stage, and the stress relaxation was explained by Narayanaswamy model at the annealing stage. However, the properties of glass under molding temperature conditions were not thoroughly studied. Mosaddegh [14] measured the viscosity and thermal expansion coefficients of N-BK7 and P-SK57 through experiments and simulation analysis, obtained the basic material parameters of the glass materials. However, the accuracy of the simulation prediction results should be further improved. Liu [15] used a thermomechanical detector to investigate the viscoelasticity of glass at high temperatures and obtained the viscoelastic parameters. These parameters were applied to simulate the glass molding process. However, the prediction results were different from the experimental measurements. Joshi [16] adopted parameter iterative method to calculate the creep curve of glass, obtained the structural relaxation parameters of BK7 and L-BAL35. However, the calculation process was complicated. Zhou [17] summarized the relationship between real stress and strain rates at different temperatures through glass molding experiments, explored different creep characteristics of high temperature glass, and optimized the thermoviscoelastic constitutive model. His study focused on the prediction of residual stress. The above mentioned studies have mainly investigated the thermoviscoelastic properties of glass, but it could provide a theoretical basis for subsequent simulation predictive analysis.

On the basis of the results of the aforementioned works, scholars have performed extensive studies on residual stress during glass molding. Tao [18] analyzed the residual stress of molded lens at the annealing stage. The results showed that the residual stress was great when the cooling rate was high. The annealing process was also optimized. Sarhadi [19] used the FORTRAN material subroutine to describe the temperature dependent viscoelasticity and structural relaxation behavior of glass materials, utilized ABAQUS software to predict residual stresses. Pallicity [20] and Yi [21] studied the influence law of residual stress on the optical properties, such as refractive index, and optimized the glass molding parameters. The above mentioned studies have mainly explored the prediction of residual stress but have failed to investigate profile deviation.

Yin [22] established finite element models for heating, molding, and annealing stages. The influence law of molding parameters, such as molding temperature, molding velocity, and annealing rate, on residual stress and profile deviation was also systematically discussed. Zhao [23] analyzed the influence of thermal expansion coefficient, molding temperature, cooling rate, and holding pressure on the profile deviation of lens. However, the thermoviscoelasticity model exhibited a low fitting accuracy. Kreilkamp [24] explored the influence of molding accuracy under non-isothermal conditions and compared it with isothermal glass molding. However, he focused mainly on the influence of temperature difference between the mold and the preform on profile deviation. Liu [25] calculated thermal shrinkage, volume change, and stress distribution and predicted the shape deviation of the molded glass optical element before performing molding experiment. The simulation results agreed well with the molding experimental results, but only single-sided aspheric lens was considered. Haq [26] determined the gradual shrinkage of glass grid in the simulation model and established a linear gradation shrinkage model to improve the profile simulation prediction model. However, double-aspheric lens was not involved.

Gurganus [27] systematically studied the optical design, mold design and manufacturing, and glass molding involved in glass molding. A free-form lens that met the requirements was also developed. Xie [28] discussed the relationship between the heating time and surface roughness of glass and analyzed the influencing factors of the molding accuracy of micro-structured array glass. Kim [29] used glass-ceramics carbon to manufacture the mold, which further improved the molding accuracy and reduced the profile deviation. Yu [30] studied ultrasonic-assisted glass molding to improve the molding accuracy. Tang [31] analyzed the glass molding of double-aspheric lens but neglected the difference in the profile deviation of the upper and lower aspheric surfaces. The above-mentioned studies have performed experiments of glass molding but have not explored the molding mechanism and profile deviation prediction of doubleaspheric lens.

The above-mentioned results are more on profile deviation during the glass molding of single-aspheric lens or microstructured optical elements and less on that of doubleaspheric lens. Double-aspheric lenses are generally prepared in production. The difference in the profile deviation of the upper and lower surfaces under the same molding condition is an urgent problem that should be addressed by enterprises to improve the accuracy of double-aspheric lens molding. Based on the thermoviscoelastic constitutive model and heat conduction theory, this study used the finite element method to establish a model for predicting the profile deviation of double-aspheric glass during molding. The influence of glass molding parameters, such as molding temperature, molding velocity, annealing rate, and holding pressure, on profile deviation was discussed, and different influence laws of the upper and lower profile deviations were analyzed. The results provide a theoretical guidance for double-spherical glass molding production.

The remainder of the study is organized as follows. Section 3 designs a concave-convex double-aspheric lens and establishes a prediction model for the profile deviation in glass molding. The thermal conduction theory and the thermoviscoelastic constitutive model are presented. Section 
4 uses finite element method to study the influence of parameters, such as molding temperature, molding velocity, annealing rate, and holding pressure, on profile deviation. The difference in the influence of same parameters on the profile deviation of concave-convex aspheric lens is also compared. The last section summarizes the conclusions.

\section{Methodology}

\subsection{Design of double-aspheric lens}

The design of the double-aspheric lens is shown in Fig. 1, where $\mathrm{S} 1$ is concave and S2 is convex, both are aspheric. The cross-sectional curve is defined by Equation (1):

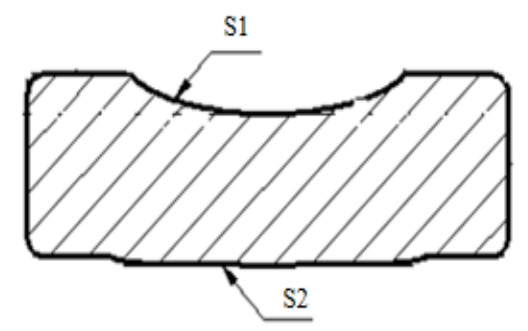

Fig.1. Design of double-aspheric glass lens

$$
\begin{aligned}
& z=f(x)=\frac{x^{2} / R}{1+\sqrt{1-(k+1)(x / R)^{2}}}+\sum_{i=2}^{n} B_{2 i} \cdot x^{2 i} \\
& \sum_{i=2}^{n} B_{2 i} \cdot x^{2 i}=A_{4} x^{4}+A_{6} x^{6}+A_{8} x^{8}+A_{10} x^{10}
\end{aligned}
$$

where $x$ is the coordinate value of curve $\mathrm{X}, z$ is the vector function of $\mathrm{X}, r$ is the curvature radius of the vertex of the curve, $\sum_{i=2}^{n} B_{2 i} \cdot x^{2 i}$ is the aspheric correction term, $k$ is the quadratic coefficient.

The parameters of the convex surface are as follows: $R=65.7 \mathrm{~mm}, \quad k=1231, \quad \mathrm{~A}_{4}=-7.851 \times 10^{-3}, \mathrm{~A}_{6}=4.1 \times 10^{-3}$, $\mathrm{A}_{8}=-2.5 \times 10^{-4}$, and $\mathrm{A}_{10}=7.3 \times 10^{-6}$. The parameters of the concave surface are as follows: $R=3.725 \mathrm{~mm}, k=0.85$, $\mathrm{A}_{4}=-2.7 \times 10^{-2}, \mathrm{~A}_{6}=8.3 \times 10^{-3}, \mathrm{~A}_{8}=1.6 \times 10^{-3}$, and $\mathrm{A}_{10}=5.6 \times 10^{-5}$.

\subsection{Simulation prediction model}

The actual environment of glass molding is relatively complicated. Thus, the environment is appropriately simplified as follows: (1) the glass preform is regarded as an incompressible material in the same direction, (2) the mold temperature distribution is uniform during the glass molding, and (3) the contact surface between the mold and the glass preform is a non-slipping boundary condition. A simulation model, as shown in Fig. 2, is established on the basis of the above-mentioned assumptions.

The simulation model adopts quadrilateral units, and the glass preform is divided into 6875 units. The upper and lower molds are divided into 1472 and 1338 units, respectively. The grid density of the glass is twice that of the mold. The generalized Maxwell model is used as the thermoviscoelastic model during the simulation. The glass is a viscoelastic body and the mold is an elastic body in the simulation model. The material properties of the glass are listed in Table 1.
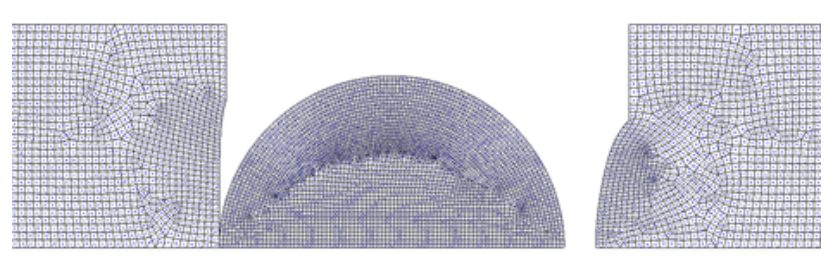

Fig. 2. Simulation prediction model of double-aspheric glass lens molding

Table 1. Thermodynamic properties of D-ZK3 optical glass

\begin{tabular}{c|c} 
Table 1. Thermodynamic properties of D-ZK3 optical glass \\
\hline Material properties & Value \\
\hline Elastic modulus $\mathrm{E}\left(\times 10^{8} \mathrm{~N} / \mathrm{m}^{2}\right)$ & 727 \\
Shear modulus $\mathrm{G}\left(\times 10^{8} \mathrm{~N} / \mathrm{m}^{2}\right)$ & 280 \\
Poisson's ratio $\mu_{0}$ & 0.299 \\
Density $\rho\left(\mathrm{kg} / \mathrm{m}^{3}\right)$ & 3700 \\
Thermal conductivity $\mathrm{k}(\mathrm{W} /(\mathrm{m} \cdot \mathrm{K}))$ & 0.78 \\
Specific heat capacity $c(\mathrm{~J} /(\mathrm{g} \cdot \mathrm{k}))$ & 0.670 \\
Transition temperature $T_{g}\left({ }^{\circ} \mathrm{C}\right)$ & 511 \\
Yield temperature $\mathrm{At}\left({ }^{\circ} \mathrm{C}\right)$ & 546 \\
Softening temperature $\mathrm{Sp}\left({ }^{\circ} \mathrm{C}\right)$ & 605 \\
\hline
\end{tabular}

\subsection{Simulation prediction theory}

In the glass molding test, the mold material is tungsten carbide J05 and the glass material is D-ZK3. The thermal boundary condition between the two materials during the molding process is expressed as [11].

$-K \frac{\partial T}{\partial n}=h_{M}\left(T-T_{M}\right)$

$-K \frac{\partial T}{\partial n}=h_{N}\left(T-T_{N}\right)$

where $k$ is the thermal conductivity of the glass, $h_{M}$ is the heat transfer coefficient between the glass and the mold, $T$ is the temperature of the glass, $h_{M}$ is the temperature of the mold, $h_{N}$ is the heat transfer coefficient between the glass and the environment; and $T_{N}$ is the temperature of nitrogen.

In general, $h_{M}$ is $2800 \mathrm{~W} /\left(m^{2} \cdot \mathrm{K}\right)$, and $h_{N}$ is $20 \mathrm{~W} /$ $\left(m^{2} \cdot \mathrm{K}\right)$.

The thermoviscoelastic constitutive model of D-ZK3 can be expressed using the generalized Maxwell model.

$$
\varepsilon(t)=\frac{\sigma_{0} w_{\infty}}{E_{\infty}}+\sigma_{0} \sum_{i=1}^{n} w_{i}\left(\frac{1}{E_{i}}+\frac{t}{\eta_{i}}\right)
$$

where $E \infty$ and $E_{\mathrm{i}}$ are the elastic moduli of the corresponding spring, $\eta_{\mathrm{i}}$ is the viscosity of the corresponding Newton sticky pot, $w_{\infty}$ and $\mathrm{w}_{\mathrm{i}}$ are the weighting factors of each unit in the generalized Maxwell model. The fitting parameters based on experimental measurement and data fitting processing are listed in Table.2. The weighting factor satisfies the following formula. 


$$
w_{\infty}+\sum_{i=1}^{n} w_{i}=1
$$

Table 2. Fitting parameters of the generalized Maxwell model

\begin{tabular}{c|c|c}
\hline Number of terms & Weighting factor & Relaxation time (s) \\
\hline 1 & 0.238 & 0.007 \\
2 & 0.238 & 0.0072 \\
3 & 0.238 & 0.0075 \\
4 & 0.238 & 0.0078 \\
5 & 0.047 & 0.001 \\
\hline
\end{tabular}

\subsection{Glass molding test}

Glass molding experiment of double-aspheric lens was performed on a multi-station glass molding machine. The experimental equipment was composed of nitrogen tank, seven-station glass molding machine, control cabinet, power switch, cooling system, and air compressor (Fig.3). The process involved seven stations, namely, preheating 1, preheating 2, preheating 3, molding, cooling 1, cooling 2, and rapid cooling.

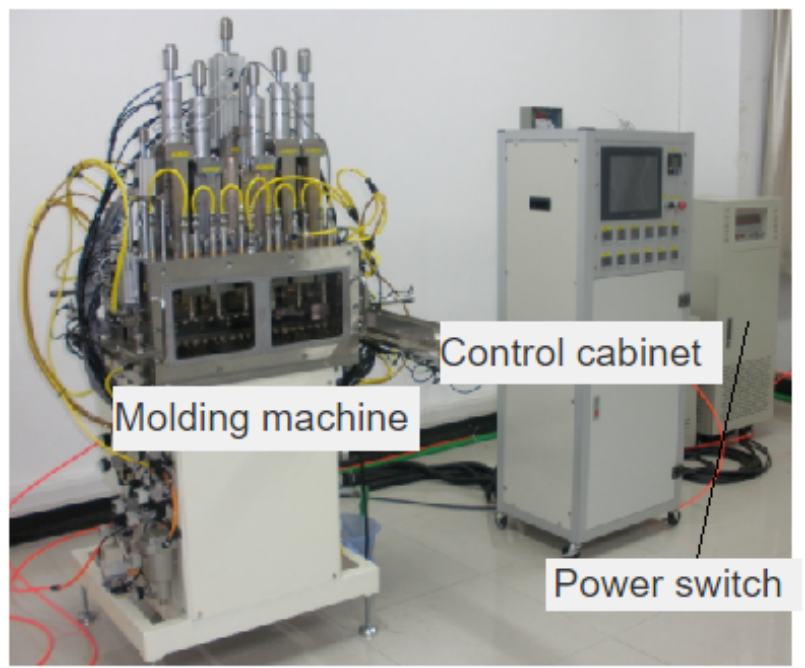

Fig. 3. Glass molding equipment

After the glass molding experiment of double-aspheric lens, the surface of the molded lens was cleaned and adhered to a customized fixture using double-sided tape. The fixed molded lens was placed on the measurement platform. After the center and measurement distance of the molded lens were adjusted, the measurement device was used to measure the surface profile (Fig.4).

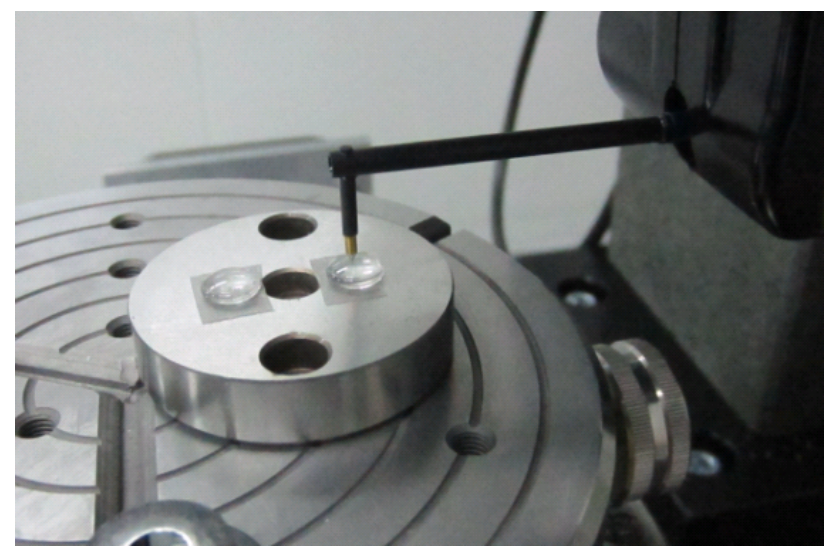

Fig. 4. Measurement of molded lens

\section{Results and Discussion}

\subsection{Influence of molding temperature on profile} deviation

The properties of glass materials are dependent on temperature. Four molding temperatures of $550{ }^{\circ} \mathrm{C}, 560{ }^{\circ} \mathrm{C}$, $570{ }^{\circ} \mathrm{C}$, and $580{ }^{\circ} \mathrm{C}$ were set to analyze the influence of molding temperature on profile deviation. The molding velocity was $0.05 \mathrm{~mm} / \mathrm{s}$, and the friction coefficient was 0.5 . The profile deviation curves of the double-aspheric upper and lower surfaces at different molding temperatures are shown in Figs. 5 and 6, respectively.

The figures show that the profile deviation of the upper and lower surfaces is the largest at the molding temperature of $550{ }^{\circ} \mathrm{C}$. The mold cavity is incompletely filled due to the poor fluidity of the preform. The maximum profile deviations of the upper and lower surfaces are $3.46 \mu \mathrm{m}$ and $1.38 \mu \mathrm{m}$, respectively. When the glass molding temperature is $570{ }^{\circ} \mathrm{C}$, the fluidity of the preform is significantly enhanced under the effect of molding pressure, and the profile deviation decreases. The maximum profile deviations of the upper and lower surfaces are $1.64 \mu \mathrm{m}$ and $0.94 \mu \mathrm{m}$, respectively. When the glass molding temperature further increases, the profile deviation increases. This result may be caused by the obvious structural relaxation of the molded lens during the annealing stage when molding temperature above $570{ }^{\circ} \mathrm{C}$, which results in a slow increase in the deviation of the profile curve. Comparing the two figures reveals that the profile deviation of the concave aspheric surface is significantly larger than that of the convex aspheric surface. At $570{ }^{\circ} \mathrm{C}$, the profile deviation of the upper surface is 1.7 times that of the lower, such difference is caused by the closer contact between the lower convex aspheric surface and the lower mold during the mold filling process. Furthermore, gravity decreases the profile deviation.

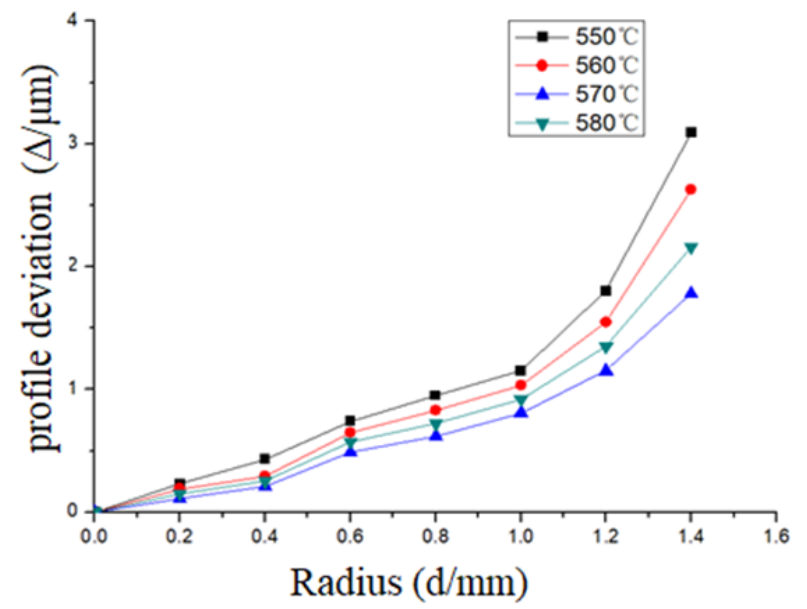

Fig. 5. Profile deviation of the concave surface under different molding temperatures

\subsection{Influence of molding velocity on profile deviation}

The molding temperature and the friction coefficient were changed to $570{ }^{\circ} \mathrm{C}$ and 0.5 , respectively. Similarly, the molding velocities were $0.02 \mathrm{~mm} / \mathrm{s}, 0.04 \mathrm{~mm} / \mathrm{s}, 0.06 \mathrm{~mm} / \mathrm{s}$, and $0.08 \mathrm{~mm} / \mathrm{s}$. Processing the simulation prediction results generates the profile deviation curves of the upper and lower surfaces with different molding velocities, as shown in Figs. 7 and 8 , respectively.

The profile deviation of the upper and lower surfaces is directly proportional to the molding velocity. When the molding velocity is $0.02 \mathrm{~mm} / \mathrm{s}$, the maximum profile deviations of the convex and concave surfaces are $0.82 \mu \mathrm{m}$ and $1.44 \mu \mathrm{m}$, respectively. When the molding velocity is 
Zhu Kejun, Shi Sheng, Chen Zhigang, Liu Zhihui and Zhang Yingying/

Journal of Engineering Science and Technology Review 13 (2) (2020)50 - 56

$0.08 \mathrm{~mm} / \mathrm{s}$, the maximum profile deviations of the convex and concave surfaces are $1.78 \mu \mathrm{m}$ and $2.51 \mu \mathrm{m}$, respectively. As the molding velocity increases with other parameters unchanged, the equivalent volume deformation of the glass from a preform to a lens is completed rapidly. The internal residual pressure also increases significantly. Accordingly, the deformation caused by structural relaxation during the annealing stage and the profile deviation increase. The profile deviation of the concave aspheric surface is significantly larger than that of the convex aspheric surface. At $0.02 \mathrm{~mm} / \mathrm{s}$, the profile deviation of the upper surface is 1.7 times that of the lower. This finding is consistent with the results of molding temperature.

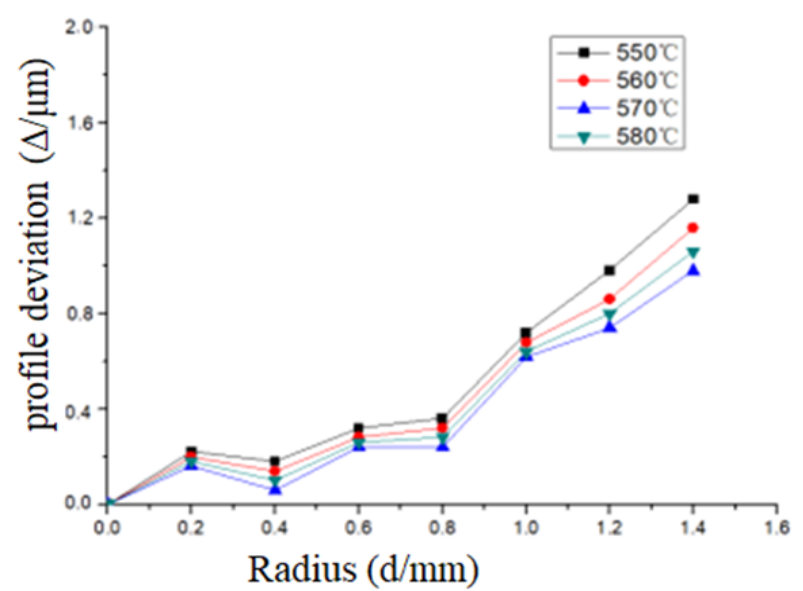

Fig. 6. Profile deviation of the convex surface under different molding temperatures

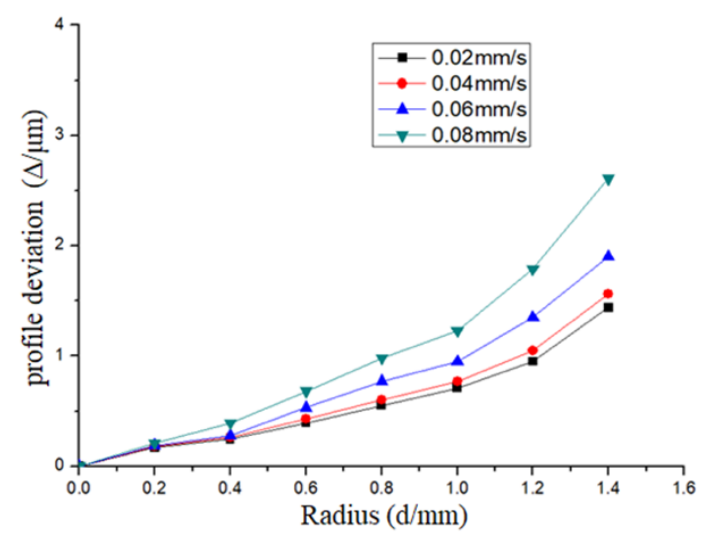

Fig. 7. Profile deviation of the concave surface under different molding velocities

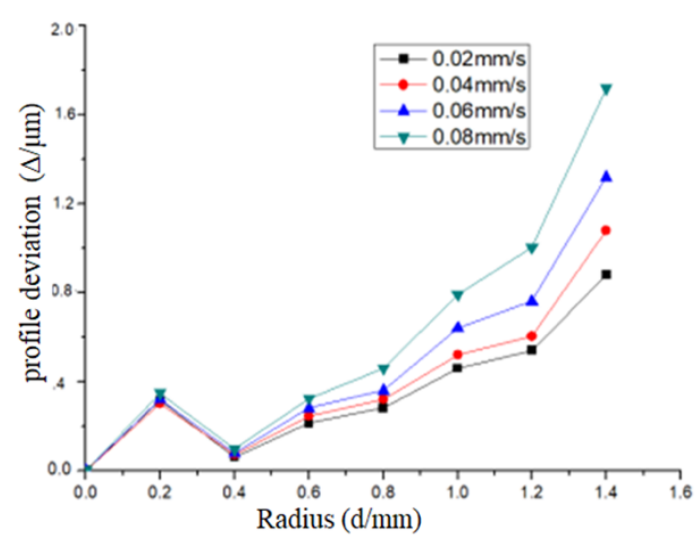

Fig. 8. Profile deviation of the convex surface under different molding velocities

\subsection{Influence of annealing rate on profile deviation}

Four different annealing rates of $0.5^{\circ} \mathrm{C} / \mathrm{s}, 1{ }^{\circ} \mathrm{C} / \mathrm{s}, 1.5^{\circ} \mathrm{C} / \mathrm{s}$, and $2{ }^{\circ} \mathrm{C} / \mathrm{s}$ were set and other parameters in each group were the same in analyzing the influence of annealing rate on profile deviation during the annealing stage. Processing the results after the annealing stage generates the profile deviation curves of the upper and lower surfaces at different annealing rates, as shown in Figs. 9 and 10, respectively.

When the annealing rate is $0.5{ }^{\circ} \mathrm{C} / \mathrm{s}$, the maximum profile deviations of the convex and concave surfaces are $1.58 \mu \mathrm{m}$ and $2.21 \mu \mathrm{m}$, respectively. At the annealing rate of $2{ }^{\circ} \mathrm{C} / \mathrm{s}$, the maximum profile deviations are $0.49 \mu \mathrm{m}$ and $1.38 \mu \mathrm{m}$, respectively. The profile deviation of the upper and lower surfaces decreases with the increase in the annealing rate. With the increase of annealing rate, the internal structure of the lens relaxes rapidly and the internal residue stress of the molded lens solidifies before being released. Thus, the profile deviation is reduced. Although increasing the annealing rate can reduce the profile deviation, but the residual stress inside the lens is large and the imaging quality is poor. Comparing the two figures reveals that the profile deviation of the concave spherical surface is significantly larger than that of the convex aspheric surface.

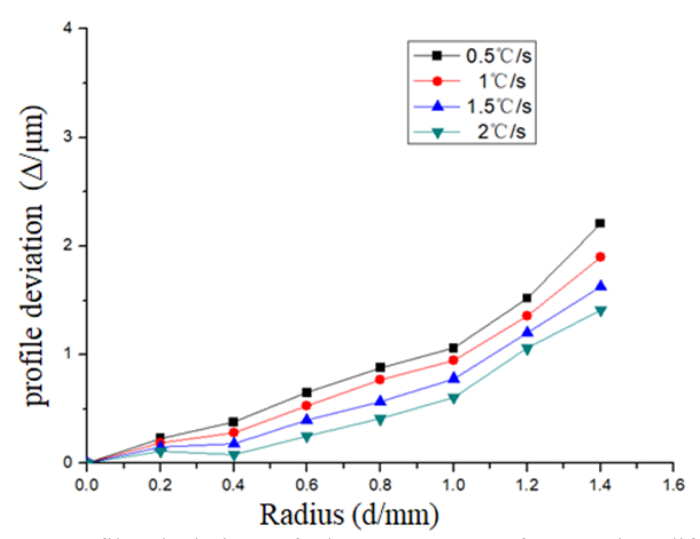

Fig. 9. Profile deviation of the concave surface under different annealing rates

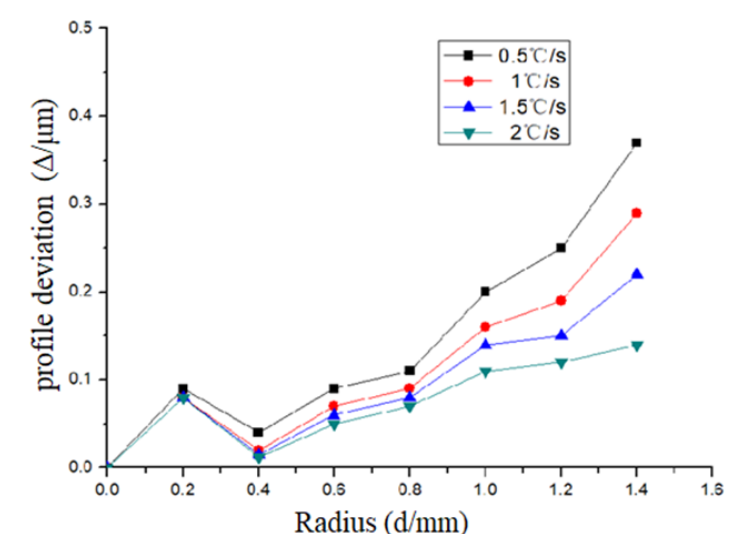

Fig. 10. Profile deviation of the convex surface under different annealing rates

4.4 Influence of holding pressure on profile deviation The holding pressures were set to $100 \mathrm{~N}, 300 \mathrm{~N}, 500 \mathrm{~N}$, and $700 \mathrm{~N}$ in analyzing the influence of the holding pressure on profile deviation at the annealing stage. The other parameters of each group were unchanged. Processing the simulation prediction results after the annealing stage generates the profile deviation curves of the upper and lower surfaces under different holding pressures, as shown in Figs. 11 and 12 , respectively. 
When the holding pressure is $100 \mathrm{~N}$, the maximum profile deviations of the convex and concave surfaces are $1.51 \mu \mathrm{m}$ and $3.27 \mu \mathrm{m}$, respectively. At the holding pressure of $700 \mathrm{~N}$, the maximum profile deviations are reduced to $0.98 \mu \mathrm{m}$ and $1.03 \mu \mathrm{m}$, respectively. The deviation of profile deviation for the upper and lower surfaces is inversely proportional to the holding pressure. As the holding pressure increases, the difference between the profile deviations of the concave and convex surfaces gradually decreases. The aspheric surfaces of the molded lens closely adhere to the cavity surface during the annealing process when the holding pressure increases. The difference in profile deviation is small when the shrinkage deformation caused by structural relaxation. Therefore, the holding pressure in the glass molding production of double-aspheric lens can be appropriately increased to reduce the difference in profile deviation. Accordingly, the overall molding accuracy can be improved, and the subsequent compensation can be facilitated.

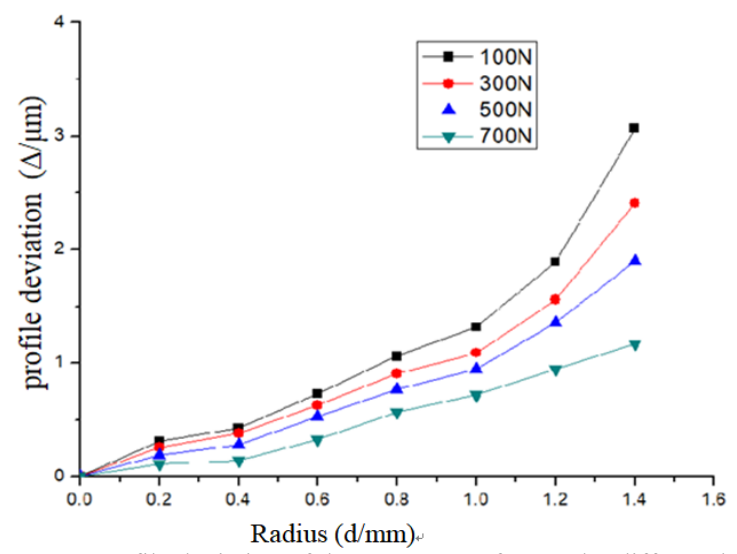

Fig. 11. Profile deviation of the concave surface under different holding pressures

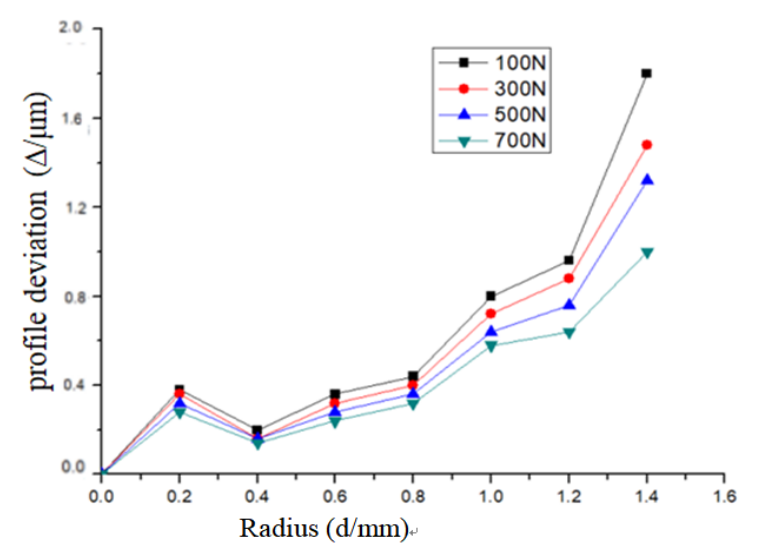

Fig. 12. Profile deviation of the convex surface under different holding pressures

\section{Conclusions}

This study established a simulation prediction model for the glass molding of double-aspheric lens based on the generalized Maxwell model to explore the influence of glass molding parameters on profile deviation. It had revealed the influence of molding parameters on the profile deviation of aspheric surfaces. The influence of molding temperature, molding velocity, annealing rate and holding pressure on profile deviation was analyzed, and the different influence laws of molding parameters on the profile deviation of upper and lower aspheric surfaces were explored. The following conclusions could be drawn:

1) When the molding temperature is lower than $570{ }^{\circ} \mathrm{C}$, the profile deviation decreases with the increase of molding temperature. However, when the molding temperature is higher than $570{ }^{\circ} \mathrm{C}$, the profile deviation gradually increases as the molding temperature increases.

2) The profile deviation of the upper and lower aspheric surfaces increases with the increase in the molding velocity.

3) The profile deviation of the upper and lower aspheric surfaces decreases with the increase in the annealing rate and the holding pressure.

4) The difference between the profile deviations of the upper and lower aspheric surfaces decreases with the increase in the holding pressure. Therefore, appropriately increasing the holding pressure can reduce the profile deviation of the upper and lower surfaces, and consequently improve the molding accuracy of double-aspheric surfaces.

By combining thermoviscoelastic theory and finite element analysis, this study explored the influence of glass molding parameters on profile deviation and analyzed the effect of molding parameters on the profile deviations of concave-convex aspheric surfaces. The results provide a reference for the production of double-aspheric lens. However, the gravity boundary conditions will be combined with this model and modified in future studies due to the lack of accurate data of gravity. Accordingly, the prediction accuracy of the upper and lower aspheric profile deviations can be enhanced.

\section{Acknowledgements}

This work was supported by the National Natural Science Foundation of China (Grant No. 91860133) and Hunan Education Department Project (Grant No. 19B516).

This is an Open Access article distributed under the terms of the Creative Commons Attribution License

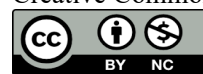

\section{References}

1. Chen, F. J., Miao, X., Tang, Y., et al, “A review on recent advances in machining methods based on abrasive jet polishing". The International Journal of Advanced Manufacturing Technology, 90(4), 2017, pp.785-799.

2. Yin, S., Jia, H., Zhang, G., et al, "Review of small aspheric glass lens molding technologies". Frontiers of Mechanical Engineering, 12(1), 2017, pp.66-76.
3. Natta, W., Duangjai, N., Rattikan, C., "A simple method using twostep hot embossing technique with shrinking for fabrication of cross microchannels on PMMA substrate and its application to electrophoretic separation of amino acids in functional drinks". Talanta, (161), 2016, pp.574-582.

4. Moore, S., Gomez, J., Lek, D., et al, "Experimental study of polymer microlens fabrication using partial-filling hot embossing technique". Microelectronic Engineering, (162), 2016, pp.57-62. 
Zhu Kejun, Shi Sheng, Chen Zhigang, Liu Zhihui and Zhang Yingying/

\section{Journal of Engineering Science and Technology Review 13 (2) (2020)50 - 56}

5. Zhang, Y., Yan, G., Li, Z., et al, "Quality improvement of collimating lens produced by precision glass molding according to performance evaluation". Optics Express, 27(4), 2019, pp.50335047.

6. Friedrichs, M., Grunwald, T., Bergs, T., "Evaluation of mold materials for precision glass molding". In: Society of Photo-Optical Instrumentation Engineers Conference Series, Teisnach, Germany: SPIE, 2019, DOI: 10.1117/12.2526769.

7. Friedrichs, M., Peng, Z., Grunwald, T., et al, "PtIr protective coating system for precision glass molding tools: Design, evaluation and mechanism of degradation". Surface and Coatings Technology, (385), 2020, pp.125378.

8. Pallicity, T. D., Vu, A. T., Ramesh, K., et al, "Birefringence measurement for validation of simulation of precision glass molding process". Journal of the American Ceramic Society, 100(10), 2017, pp.4680-4698.

9. Jain, A., Yi A., "Finite element modeling of structural relaxation during annealing of a precision molded glass lens". Journal of Manufacturing Science and Engineering, 128(3), 2006, pp.683-690.

10. Liu, Y., Xing, Y., Yang, C., et al, "Simulation of heat transfer in the progress of precision glass molding with a finite element method for chalcogenide glass". Applied optics, 58(27), 2019, pp.73117318.

11. Zhou, T., Zhu, Z., Liu, X., et al, "A review of the precision glass molding of chalcogenide glass for infrared optics". Micromachines, 9(7), 2018, DOI: 10.3390/mi9070337

12. Su, L. J., Wang, F., He, P., et al, "An integrated solution for mold shape modification in precision glass molding to compensate refractive index change and geometric deviation". Optics and Lasers in Engineering, 53(1), 2014, pp.98-103.

13. Saotome, Y., Kenichi, I., Narihito, S., "Micro-formability of optical glasses for precision molding". Journal of Materials Processing Technology, (140), 2003, pp.379-384.

14. Mosaddegh, P., Ziegert, J., "The effect of temperature on the stickslip friction behavior of optical glasses in precision glass molding". Applied Mechanics \& Materials, (307), 2013, pp.381-386.

15. Liu, W., Shen, P., "Viscoelastic properties of chalcogenide glasses and simulation of molding process". Infrared and Laser Engineering, (03), 2012, pp.569-574.

16. Joshi, D., Mosaddegh, P., Musgraves, J. D., et al, "Thermomechanical characterization of glass at high temperature using the cylinder compression test. Part I: Viscoelasticity, friction, and PPV”. Journal of Rheology, 57(5), 2013, DOI: 10.1122/1.4817434.

17. Yan, J., Zhou, T., Masuda, J., et al, "Modeling high-temperature glass molding process by coupling heat transfer and viscous deformation analysis". Precision Engineering, 33(2), 2009, pp.150159.

18. Tao, B., Shen, L. G., Yi, A., et al, "Reducing refractive index variations in compression molded lenses by annealing". Optics and Photonics Journal, (3), 2013, pp.118-121.
19. Sarhadi, A., Hattel J. H., Hansen, H. N., "Precision glass molding: validation of an FE model for thermo-mechanical simulation". International Journal of Applied Glass Science, 5(3), 2014, pp.297312.

20. Pallicity, T. D., Vu, A. T., Ramesh, K., et al, "Birefringence measurement for validation of simulation of precision glass molding process". Journal of the American Ceramic Society, 100(10), 2017, pp.4680-4698.

21. Yi, A., Tao, B., Klocke, F., et al, "Residual stresses in glass after molding and its influence on optical properties". Procedia Engineering, (19), 2011, pp.402-406.

22. Yin, S., Zhu, K., Yu, J., et al, "Micro aspheric glass lens molding process". Journal of Mechanical Engineering, (58)15, 2012, pp.182-191.

23. Zhao, W., Chen, Y., Shen, L., et al, "Investigation of the refractive index distribution in precision compression glass molding by use of 3D tomography". Measurement Science and Technology, 20(5), 2009, DOI: 10.1088/0957-0233/20/5/055109.

24. Kreilkamp, H., Vu, A. T., Dambon, O., et al, "Nonisothermal glass molding of complex LED optics". In: 77th Conference on Glass Problems: A Collection of Papers Presented at the 77th Conference on Glass Problems, Columbus, USA: CESP, 2016, pp.141-149.

25. Liu, G., Vu, A. T., Dambon, O., et al, "Glass material modeling and its molding behavior". MRS advances, 2(16), 2017, pp.875-885.

26. Haq, M. R., Kim, J., Ju, J., et al, "Development of shrinkage model of micro structured vitreous carbon mold for glass molding". Journal of Mechanical Science and Technology, 33(12), 2019, pp.5747-5752.

27. Gurganus, D., Novak, S., Symmons A., et al, "Precision glass molding of freeform optics". In: Optical Fabrication and Testing, Optical Society of America, California, USA: SPIE, 2018, DOI: 10.1117/12.2320574

28. Xie, J., Zhou, T., Ruan, B., et al, "Effects of interface thermal resistance on surface morphology evolution in precision glass molding for microlens array". Applied optics, 56(23), 2017, pp. 6622-6630.

29. Kim, Y. K., Ju, J. H., Kim, S. M., "Replication of a glass microlens array using a vitreous carbon mold". Optics express, 26(12), 2018, pp.14936-14944.

30. Yu, Q., Zhou, T., Jiang, Y., et al, "Preparation of grapheneenhanced nickel-phosphorus composite films by ultrasonic-assisted electroless plating”. Applied Surface Science, (435), 2018, pp.617625 .

31. Tang, K., Kong, M., Zhu, Y., et al, "Experimental study on precision molding of small dual aspherical chalcogenide glass lens". Infrared and Laser Engineering, 47(4), 2018, DOI: 10.3788/IRLA201847.0418006 